\title{
Size-controlled growth of nanoparticles in a highly ionized pulsed plasma
}

\author{
Iris Pilch, Daniel Söderström, N Brenning and Ulf Helmersson
}

\section{Linköping University Post Print}

N.B.: When citing this work, cite the original article.

Original Publication:

Iris Pilch, Daniel Söderström, N Brenning and Ulf Helmersson, Size-controlled growth of nanoparticles in a highly ionized pulsed plasma, 2013, Applied Physics Letters, (102), 3, . http://dx.doi.org/10.1063/1.4788739

Copyright: American Institute of Physics (AIP) http://www.aip.org/

Postprint available at: Linköping University Electronic Press http://urn.kb.se/resolve?urn=urn:nbn:se:liu:diva-89521 


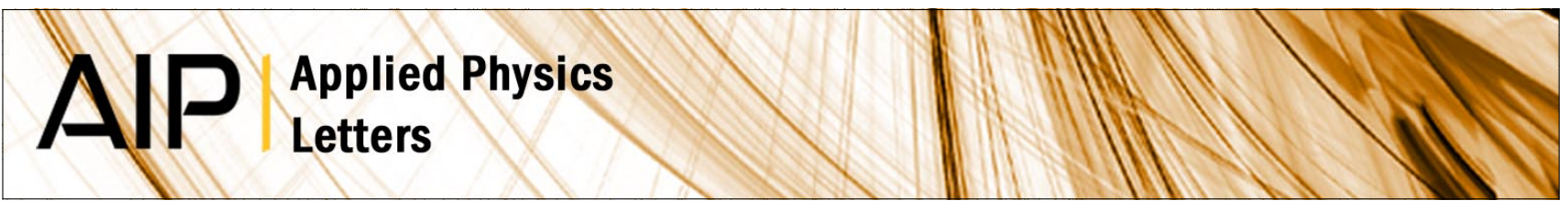

Size-controlled growth of nanoparticles in a highly ionized pulsed plasma

I. Pilch, D. Söderström, N. Brenning, and U. Helmersson

Citation: Appl. Phys. Lett. 102, 033108 (2013); doi: 10.1063/1.4788739

View online: http://dx.doi.org/10.1063/1.4788739

View Table of Contents: http://apl.aip.org/resource/1/APPLAB/v102/i3

Published by the American Institute of Physics.

\section{Related Articles}

Atomic structure of tensile-strained $\mathrm{GaAs} / \mathrm{GaSb}(001)$ nanostructures

Appl. Phys. Lett. 102, 102105 (2013)

Fabrication and optical properties of large-scale arrays of gold nanocavities based on rod-in-a-tube coaxials Appl. Phys. Lett. 102, 103103 (2013)

The induction of nanographitic phase on Fe coated diamond films for the enhancement in electron field emission properties

J. Appl. Phys. 113, 094305 (2013)

Note: Size effects on the tensile response of top-down fabricated Si nanobeams

Rev. Sci. Instrum. 84, 036102 (2013)

Double-sided tin nanowire arrays for advanced thermal interface materials

Appl. Phys. Lett. 102, 093105 (2013)

\section{Additional information on Appl. Phys. Lett.}

Journal Homepage: http://apl.aip.org/

Journal Information: http://apl.aip.org/about/about_the_journal

Top downloads: http://apl.aip.org/features/most_downloaded

Information for Authors: http://apl.aip.org/authors

\section{ADVERTISEMENT}

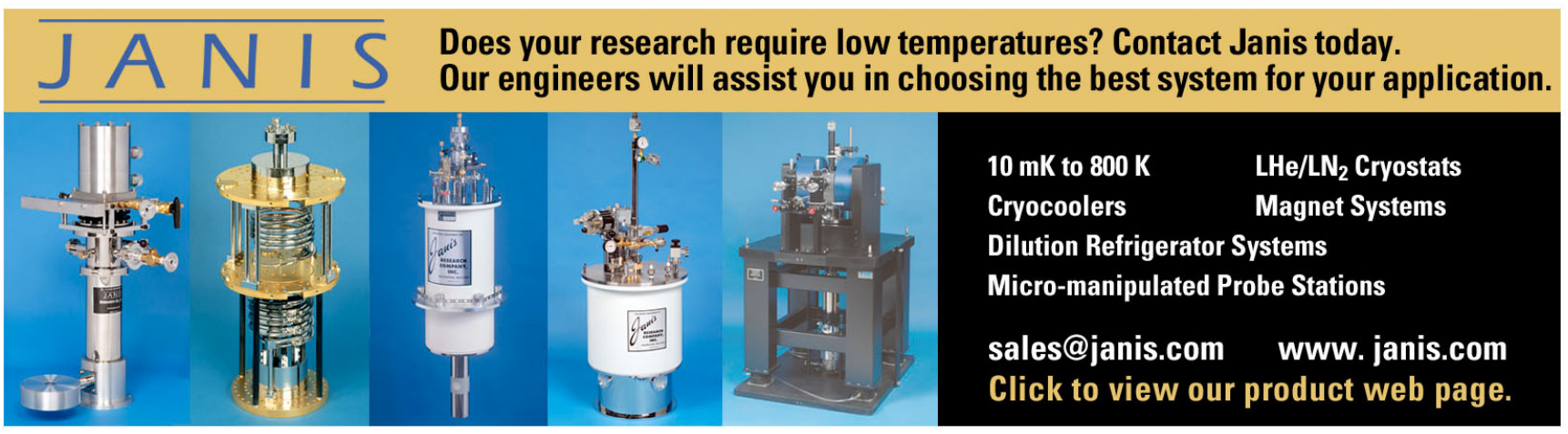




\title{
Size-controlled growth of nanoparticles in a highly ionized pulsed plasma
}

\author{
I. Pilch, ${ }^{1, a)}$ D. Söderström, ${ }^{1}$ N. Brenning, ${ }^{2}$ and U. Helmersson ${ }^{1}$ \\ ${ }^{1}$ Plasma \& Coatings Physics Division, IFM-Materials Physics, Linköping University, SE-581 83 Linköping, \\ Sweden \\ ${ }^{2}$ Division of Space \& Plasma Physics, School of Electrical Engineering, Royal Institute of Technology, \\ SE-10 044 Stockholm, Sweden
}

(Received 14 November 2012; accepted 4 January 2013; published online 24 January 2013)

\begin{abstract}
Copper nanoparticles (NPs) were synthesized using a high power pulsed hollow cathode technique and the produced NPs were studied as a function of pulse parameters, i.e., frequency, peak current, and pulse width. It was found that the particle size can be altered in a range from 10 to $40 \mathrm{~nm}$ by changing any one of the pulse parameters. The mechanisms of NP synthesis with respect to a pulsed discharge and a high degree of ionization of the sputtered material are discussed. (c) 2013 American Institute of Physics. [http://dx.doi.org/10.1063/1.4788739]
\end{abstract}

Nanoparticles (NPs) are of interest in many applications due to their physical and chemical properties that greatly differ from bulk properties. Examples include catalytic effects because of their high surface-to-volume ratio, ${ }^{1,2}$ and interaction with light due to plasmonic activity. ${ }^{3,4}$ For both examples, the response is a function of NP size and the geometry of the NP. Therefore, a technique for controlling these parameters to accurately synthesize NPs-in sufficient number and without agglomeration-is desired.

One method for synthesizing NPs is by using plasma discharges where the material for NP growth is supplied to the plasma via sputtering the source material from a target. ${ }^{5-7} \mathrm{~A}$ benefit of this method is that NPs of any material, which can be sputtered, can in principle be synthesized. When the NPs have grown to a certain size, they attain a negative charge. ${ }^{8,9}$ The electrostatic repulsion between such charged NPs hinders further agglomeration inside the plasma. Using dc discharges, it has been shown that the size of the NPs can be varied by changing process parameters such as gas flow, gas pressure, and discharge power. ${ }^{10-12}$ As a way of obtaining additional control parameters, pulsing has been used in the related field of magnetron sputtering for thin film growth, where it has been shown to give a wider accessible range of plasma parameters. ${ }^{13}$ Most important in the present application is that the momentary density of sputtered material can be increased in a pulse while keeping the gas temperature low, and that a high degree of ionization of the sputtered material can be achieved. ${ }^{14}$ Nanoparticle synthesis using pulsed plasma discharges is a growing research field. ${ }^{15-17}$ For instance, Stranák et al. ${ }^{15}$ demonstrated particle size control -8 to $10 \mathrm{~nm}$ in diameter-in a pulsed magnetron over a frequency range from 0.1 to $25 \mathrm{kHz}$. In this paper, we report a NP synthesis method that makes use of a high power pulsed hollow cathode (HC) discharge, a technique that can exploit a high degree of ionization of the sputtered species. We demonstrate that it is possible to tailor the size of NPs with spherical shape and diameters in the range of 10 to $40 \mathrm{~nm}$ by varying the pulse frequency, the pulse amplitude, and the pulse length. As a special case, we demonstrate that size control is achievable with constant average power.

${ }^{\text {a)} E l e c t r o n i c ~ m a i l: ~ i r i p i @ i f m . l i u . s e . ~}$
The NPs are formed by sputtered vapor in the gas phase, and the growth can be divided into three main stages: ${ }^{9}$ nucleation, coagulation, and accretion by attachment of atoms and ions. The nucleation starts when two metal particles (atoms or ions) form a dimer by a three-body collision with a third particle. These dimers then grow by attachment of individual metal atoms and ions to small clusters. In the second stage, the density of such clusters has become large enough for coagulation between them. The small clusters have a charge that is determined by a stochastic process and they can be neutral, positively, or negatively charged. However, when the clusters attain a certain size, of the order of a few to ten nanometers, ${ }^{9,18}$ the charge becomes determined by the balance of ion and electron currents to the cluster, i.e., the floating condition, and the clusters approach the floating potential, ${ }^{19,20}$

$$
U_{\mathrm{fl}}=-K_{1} \frac{k_{\mathrm{B}} T_{\mathrm{e}}}{e},
$$

where $k_{\mathrm{B}}$ is the Boltzmann constant, $e$ the elementary charge, and $T_{\mathrm{e}}$ the electron temperature. The factor $K_{1}$ depends on the ion mass and temperature ratio of electrons and ions, but can be assumed to be a constant factor of 2.41 for $T_{\mathrm{e}} / T_{\mathrm{i}} \approx 100{ }^{8}$ Nanoparticles that attained floating potential repel each other, further growth by coagulation is suppressed and stage three begins with growth only by attachment of metal atoms and ions. For the atoms, this process is independent of the charge and their attachment cross section is given by the geometrical cross section of the NP,

$$
\sigma_{\text {att,atom }}=\pi R_{n}^{2},
$$

where $R_{n}$ is its radius. The attachment cross section for ions with opposite charge to the NP is given by, ${ }^{19,21}$ $\sigma_{\text {att,ion }}=\pi R_{n}^{2}\left(1+\left|e U_{\mathrm{fl}}\right| / E_{\mathrm{kin}}\right)$, where $E_{\mathrm{kin}}$ is the kinetic energy of ions that is of the order of $k_{\mathrm{B}} T_{\mathrm{i}}$ for low drift velocities. Combining these expressions with the floating potential gives the effective collection cross section for singly charged, thermally distributed ions as,

$$
\sigma_{\text {att,ion }}=\pi R_{n}^{2}\left(1+K_{1} \frac{T_{\mathrm{e}}}{T_{\mathrm{i}}}\right) .
$$


For typical discharge parameters, e.g., $T_{\mathrm{e}}=2 \mathrm{eV}$ and $T_{\mathrm{i}}=0.03 \mathrm{eV}$, this is more than two orders of magnitude larger than the atom attachment cross section, Eq. (2).

By varying the plasma parameters (sputtered density and degree of ionization, gas temperature, and electron temperature), there are two possibilities to influence the NP growth. In the first two stages of nucleation and coagulation, the most important parameters are the density of sputtered material and the gas temperature, the latter because a high gas temperature suppresses nucleation. ${ }^{22,23}$ In the third stage of growth, the most important parameters are the degree of ionization of the sputtered species and the electron temperature. In an environment with a given number density of sputtered material, variations in these two parameters-from the difference in the attachment rates due to different crosssections [Eqs. (2) and (3)] — can influence the rate of NP growth by several orders of magnitude.

For achieving a plasma in which the above identified key discharge parameters can be flexibly varied, a setup was developed using a hollow cathode in pulsed operation. Due to the hollow cathode effect, a high degree of ionization can be achieved. ${ }^{24}$ The pulsed technique applied to the hollow cathode is similar to high power impulse magnetron sputtering, ${ }^{25}$ which has shown to increase the degree of ionization substantially as compared to dc magnetron sputtering. ${ }^{14}$ Further, the small orifice of the hollow cathode leads to an ejection of the sputtered source vapor into a smaller region and maintains a higher material density.

A sketch of the experimental setup is shown in Fig. 1(a). The chamber is cylindrical with a length of $430 \mathrm{~mm}$ and a diameter of $290 \mathrm{~mm}$. The target is a hollow cathode made of copper which is mounted in the center of the lid. The cathode (length: $54 \mathrm{~mm}$, inner diameter: $5 \mathrm{~mm}$ ) is water-cooled. A grounded anode ring (AR, diameter: $30 \mathrm{~mm}$ ) is placed $45 \mathrm{~mm}$
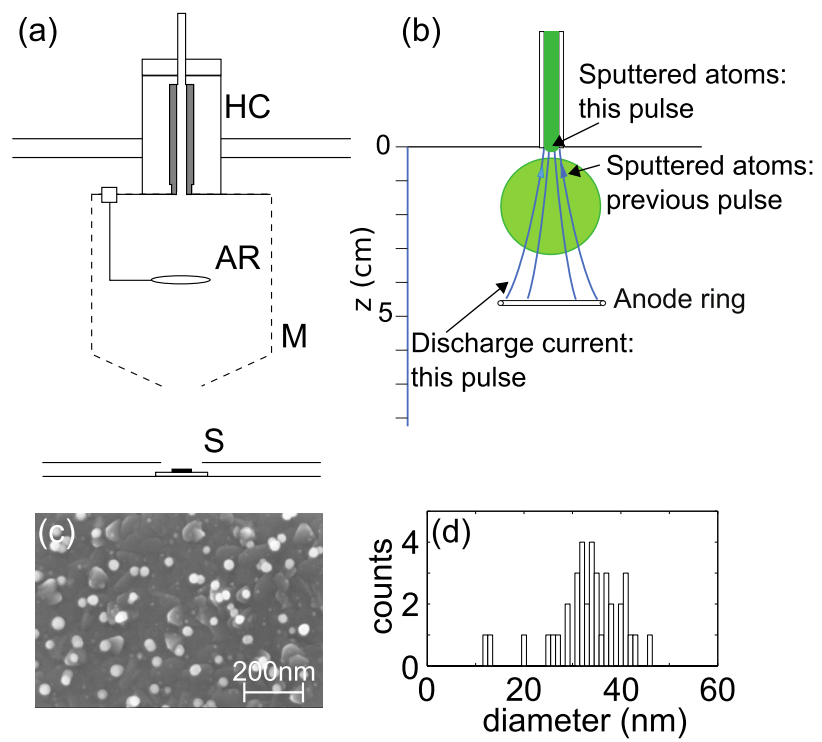

FIG. 1. (a) Sketch of the setup. The gas is fed through the hollow cathode (HC). The hollow cathode is biased and a grounded anode ring (AR) is placed below the hollow cathode and enclosed by a mesh (M). The NPs are collected on a positively biased substrate (S). (b) Sketch of the sputtered metal atoms ejected during consecutive pulses. (c) Example of a SEM planview micrograph of spherical NPs. The structure of the titanium film can be seen as plates sticking out of the surface. (d) The corresponding size distribution calculated from the micrograph. below the cathode. The motivation for placing the anode ring in this location is to force the discharge current through the region in which the NPs grow [Fig. 1(b)], thus providing a means to influence the plasma parameters during the growth process. A stainless steel mesh cage encloses the anode ring. The substrate table is made of stainless steel and can be loaded with six substrates. The distance between the substrate table and the cathode is $160 \mathrm{~mm}$. The substrates have a size of $10 \times 10 \mathrm{~mm}$ and are made of silicon coated with a $200 \mathrm{~nm}$ thick titanium layer; they were positively biased at $10 \mathrm{~V}$ during the experiments. The base pressure of the chamber was $5.3 \times 10^{-4} \mathrm{~Pa}$, and argon (99.9999\%) at a pressure of $107 \mathrm{~Pa}$ was used as the working gas. The pulsed parameters were varied for the frequency in a range between 250 and $1300 \mathrm{~Hz}$ and the pulse width between 10 and $77 \mu \mathrm{s}$; a peak current of up to $20 \mathrm{~A}$ was used.

The NPs were analyzed using a scanning electron microscope (SEM, LEO 1550 Gemini) by taking a number of planview micrographs with different magnifications at several positions on the substrate. From each micrograph, the nanoparticle sizes were determined and the measured size distributions were combined to improve the statistics. The mean size and the standard deviation were determined by fitting a lognormal distribution. An example of a micrograph is given in Fig. $1(\mathrm{c})\left[f=698 \mathrm{~Hz}, I_{\mathrm{H}}=10.2 \mathrm{~A}\left(U_{\mathrm{H}}=576 \mathrm{~V}\right)\right.$, and $\left.t_{\mathrm{PW}}=30 \mu \mathrm{s}\right]$ together with the corresponding size distribution determined from the micrograph, Fig. 1(d). The NPs are the whiter features that have a spherical shape. Surface features from the titanium coating of the substrate are visible as plates sticking out of the surface.

The dynamics of the atom and ion motion in this type of discharge has recently been studied by means of simulations by Hasan $e t a l .^{26}$ The general behavior can be summarized as follows: Initially, a puff containing sputtered material is ejected with each pulse, see Fig. 1(b). The metal atoms form one cloud, which drifts with the gas flow and expands by classical diffusion. At the time of a pulse, the atom cloud from the previous pulse is typically between the hollow cathode and the anode ring, and has a diameter of a few $\mathrm{cm}$. The ejected atom cloud will then partially overlap with the remains of the atom clouds from the previous pulses, i.e., the fractions that have not been consumed in the NP growth process. The ions form a separate cloud [not shown in Fig. 1(b)] that is subject to ambipolar diffusion, a much faster process than the classical diffusion of the metal atoms. When a pulse is applied, the center of the ion cloud from the previous pulse is, therefore, typically below the anode ring. Both for neutral and ionized sputtered material, there is thus a direct interaction of overlapping sputtered material from subsequent pulses. The clouds containing neutrals merge with each other, and the ions ejected in subsequent pulses flow through these overlapping neutral clouds providing new material. In addition, there is an indirect interaction when the discharge current of subsequent pulses penetrates the region between the hollow cathode and the anode ring. This can affect both the degree of ionization of the neutral material and, through the electron temperature [see Eq. (3)], the growth rate of NPs in the attachment phase.

Consider a typical NP which is initiated by the formation of a dimer in the ejected puff of sputtered material, at a 
time when it is close to the cathode opening. Assuming that in its subsequent trajectory, it moves with the gas as modeled by Hasan et al., ${ }^{26}$ and will-during the first $\mathrm{ms}$ - be pushed 10 to $20 \mathrm{~mm}$ from the cathode by the fast gas flow associated with the puff. Approximately at this distance, it will then be picked up by the slower steady feed gas flow pattern and take an additional 10 to $15 \mathrm{~ms}$ to reach the anode ring. For the frequency $f=700 \mathrm{~Hz}$, the NP will be passed by typically ten ejected ion clouds, and also becomes exposed to ten discharge current pulses with enhanced ionization and elevated electron temperature. The densities of the sputtered metal atom and ion clouds will, due to the NP growth itself, be reduced by an unknown fraction below those modeled in Ref. 26. We are, therefore, limited to qualitative analysis and identify four mechanisms that would favor the growth of NPs: (1) Pulse strength: strong pulses will give a higher density of single-pulse sputtered material close to the cathode. This enhances the two first phases of nucleation and coagulation. This also holds true for (2) neutral overlap: mixing with the atom clouds from the previous and subsequent pulse(s). (3) Ion overlap: we define as NPs being swept over by the ion clouds from subsequent pulses. This makes the faster ion attachment to NPs possible in the third phase of growth, and this, in turn, is enhanced by an increase in electron temperature due to (4) discharge overlap: penetration of the discharge current from subsequent pulses. Below we present four experiments demonstrating that the trends of NP sizes with process parameters $\left(f, I_{\mathrm{H}}\right.$, and $\left.t_{\mathrm{PW}}\right)$ can all be qualitatively understood in terms of these four mechanisms.

In the first two experiments, the pulse amplitude and the pulse length were varied, while the pulse frequency was kept constant at $f=700 \mathrm{~Hz}$. As described, most of the drift time of a NP down to the anode ring is spent in the feed gas flow which is almost independent of the pulse strength. Therefore, a NP is exposed to approximately the same number of pulses in the overlap mechanisms.

Figure 2(a) shows the effect of varying the peak current. Two size populations are found. At the highest current, still a tiny fraction of smaller NPs is produced; this quantity is too small to obtain reliable statistics for determining a size distribution. For the dominating population of NP size data, there is an approximately linear relation between the size and the peak current. The sputter yield can be assumed to be constant which allows also to assume a linear increase of the amount of sputtered material with the peak current. The variation in
NP size with peak current is, therefore, proposed to be mainly a pulse strength effect due to the amount of material available for growth. However, with stronger pulses there will also be a stronger discharge overlap, and possibly also a higher degree of ionization. Both effects would enhance NP growth in the third phase.

Figure 2(b) shows the effect of varying the pulse width $t_{\mathrm{PW}}$. The peak current was kept constant by adjusting the discharge voltage in the range $740 \mathrm{~V}\left(t_{\mathrm{PW}}=77 \mu \mathrm{s}\right)$ to $472 \mathrm{~V}$ $\left(t_{\mathrm{PW}}=10 \mu \mathrm{s}\right)$. The combined effect of longer pulse width, constant peak current, and lower applied voltage is that the amount of sputtered material increases, but slower than linearly with the pulse width. This is consistent with the trend in size in Fig. 2(b) which, therefore, is proposed to be primarily a pulse strength effect. The fraction of small NPs disappears, and instead the width of the size distribution becomes broader as compared to Fig. 2(a). Such broadening of the size distribution could be explained by having a situation where different stages of the NP growth may take place at the same time. This could be the case when assuming that the ejected cloud is more spread out for longer pulses and, hence, the material density might be less concentrated.

In both aforementioned experiments, the pulse strength effect is proposed to be the primary mechanism to describe the trends in NP size. We stress that this does not mean that we propose the three types of overlap to be inconsequential, only that we do not see them as directly linked to the size trends. The effect of overlaps is more directly studied by changing the frequency, shown in Fig. 2(c). The model calculations $^{26}$ show that the production, the degree of ionization, and the extraction of sputtered material in each individual pulse are only minimally influenced by the frequency, apart from a minor interaction between pulses for the highest frequencies in the studied range. Still, it was possible to vary the NP size in the range 10 to $40 \mathrm{~nm}$. This is a clear demonstration that there must be some kind of interaction between the pulses-i.e., some type of overlap(s) because the amount of sputtered material in each pulse is constant. The "neutral overlap" of metal atom clouds increases with the frequency, which would enhance the formation of dimers and small clusters. "Ion overlap" also increases since a larger number of metal ion clouds pass by the NP growth region delivering new material. Further, a NP in the third stage of growth will be subject to a number of "discharge overlaps" in which the ion attachment efficiency
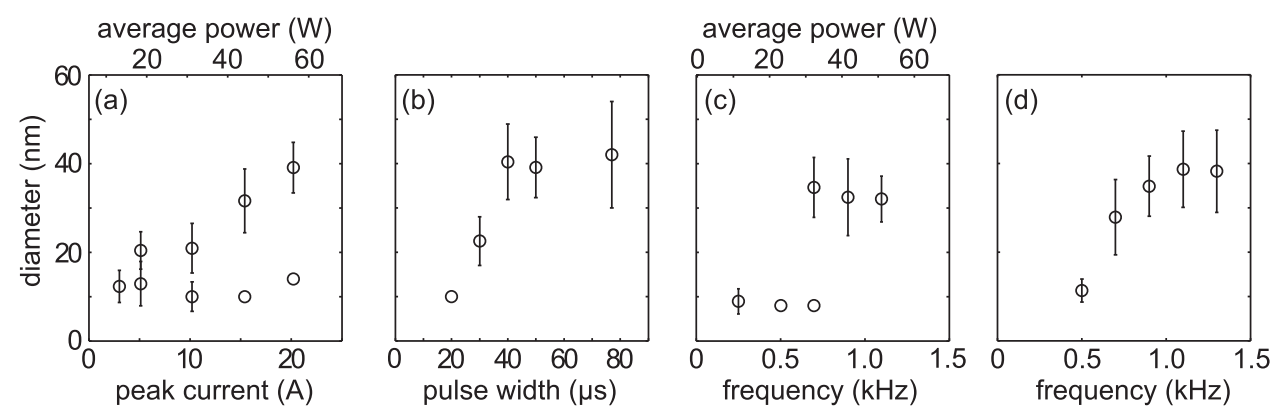

FIG. 2. Mean NP diameters obtained with the pulsed hollow cathode for varying process parameters of (a) peak current $\left(f=700 \mathrm{~Hz}, t_{\mathrm{PW}}=30 \mu \mathrm{s}\right)$, (b) pulse width $\left(f=700 \mathrm{~Hz}, I_{\mathrm{H}}=10 \mathrm{~A}\right)$, (c) frequency at constant energy per pulse $\left(I_{\mathrm{H}}=10 \mathrm{~A}, t_{\mathrm{PW}}=30 \mu \mathrm{s}\right)$, and $(\mathrm{d})$ frequency at constant average power $\left(P_{\mathrm{av}}=30 \mathrm{~W}, t_{\mathrm{PW}}=30 \mu \mathrm{s}\right)$. The peak current was adjusted to keep the average power constant with frequency. The error bar indicates the standard deviation of the size distribution and for points with no error bar the statistic was too poor to estimate it. 
could increase dramatically for negatively charged NPs. With several independent mechanisms, the combination is likely to give a size growth faster than a linear $f$-trend, at least up to the point where most of the sputtered material is consumed for NP formation. The fast rise and saturation in NP size, seen around $700 \mathrm{~Hz}$ in Fig. 2(c), might be caused by such effects.

Figure 2(d) shows an example where the NPs are varied at constant average power. In this case, the pulse length is kept constant at $30 \mu \mathrm{s}$, while the frequency and peak current were varied to give a constant average power $P_{\mathrm{av}}=30 \mathrm{~W}$. This involves two opposing trends and two counteracting mechanisms. With increasing frequency, we have the increasing size trend in Fig. 2(c). Higher frequency, however, is now compensated by a lower peak current which, according to Fig. 2(a), would give smaller NPs. Figure 2(d) shows that these two effects do not cancel. A variation in NP size is possible over the full range from 10 to $40 \mathrm{~nm}$, and with several attractive features: a smooth size variation rather than the step-like function in Fig. 2(c), a smaller spread in size than in Fig. 2(b), and without the two-size distribution in Fig. 2(a).

It has been found that the NP size can be adjusted in a range between 10 and $40 \mathrm{~nm}$ by using a high power pulsed hollow cathode and altering the process conditions. It was found that the NP size can be controlled by adjusting one of the pulse parameters, i.e., frequency, pulse width, or peak current. In these cases, the NP size always increases with average discharge power. In addition, it was shown that the NPs size could be controlled also when keeping the average power constant, by changing the frequency and adjusting the peak current. The growing NPs are transported together with the gas flow and, hence, in a cloud of metal atoms which drift with the background gas, and expands by diffusion. Subsequent neutral clouds of this type overlap, and are also periodically flushed by ion clouds as the ion ambipolar diffusion takes place on a faster time scale than neutral convection and diffusion. Four basic mechanisms affecting the growth dynamics due to the pulsing of the discharge are proposed, which provide control parameters for the growth: pulse strength, neutral overlap, ion overlap, and discharge overlap. The pulse strength determines the amount of material sputtered in each pulse, while the frequency is the most important pulse parameter to influence the degree of overlaps between the pulses. A high degree of ionization is a key feature in the process since it is expected to dramatically increase the growth rate for NP growth as soon as the NPs become negatively charged.

The authors would like to thank D. Magnfält and R. Gunnarsson for experimental contributions and M. I. Saadeh for helpful discussions on modeling. The work was financially supported by the Swedish Research Council under Grant No. 349-2008-6582 through the Linköping Linneaus Environment LiLi-NFM and by a grant for strategic initiatives by Linköping University.

${ }^{1}$ B. R. Cuenya, Thin Solid Films 518, 3127 (2010).

${ }^{2}$ A. Z. Moshfegh, J. Phys. D 42, 233001 (2009).

${ }^{3}$ S. Linic, P. Christopher, and D. B. Ingram, Nat. Mater. 10, 911 (2011).

${ }^{4}$ E. A. Coronado, E. R. Encina, and F. D. Stefani, Nanoscale 3, 4042 (2011).

${ }^{5}$ H. Hahn and R. S. Averback, J. Appl. Phys. 67, 1113 (1990).

${ }^{6} \mathrm{H}$. Haberland, M. Karrais, M. Mall, and Y. Thurner, J. Vac. Sci. Technol. A 10, 3266 (1992).

${ }^{7}$ K. Ishii, J. Vac. Sci. Technol. A 7, 256 (1989).

${ }^{8}$ A. Piel, Plasma Physics: An Introduction to Laboratory, Space, and Fusion Plasmas, 1st ed. (Springer, 2010), Chap. 10.

${ }^{9}$ M. A. Lieberman and A. J. Lichtenberg, Principles of Plasma Discharges and Materials Processing, 2nd ed. (John Wiley \& Sons, Hoboken, New Jersey, 2005), Chap. 17.4, pp. 662-668

${ }^{10}$ M. Gracia-Pinilla, E. Martínez, G. S. Vidaurri, and E. Pérez-Tijerina, Nanoscale Res. Lett. 5, 180 (2009).

${ }^{11}$ H. Sakuma, H. Aoshima, and K. Ishii, J. Magn. 11, 103 (2006).

${ }^{12}$ A. I. Ayesh, N. Qamhieh, H. Ghamlouche, S. Thaker, and M. El-Shaer, J. Appl. Phys. 107, 034317 (2010).

${ }^{13}$ J. T. Gudmundsson, N. Brenning, D. Lundin, and U. Helmersson, J. Vac. Sci. Technol. A 30, 030801 (2012).

${ }^{14}$ J. Bohlmark, J. Alami, C. Christou, A. Ehiasarian, and U. Helmersson, J. Vac. Sci. Technol. A 23, 18 (2005).

${ }^{15}$ V. Stranák, S. Block, S. Drache, Z. Hubicka, C. A. Helm, L. Jastrabík, M. Tichý, and R. Hippler, Surf. Coat. Technol. 205, 2755 (2011).

${ }^{16}$ R. Werner, T. Höche, and S. G. Mayr, Cryst. Eng. Comm. 13, 3046 (2011).

${ }^{17}$ I. Matsui, J. Nanopart. Res. 8, 429 (2006).

${ }^{18}$ C. Cui and J. Goree, IEEE Trans. Plasma Sci. 22, 151 (1994).

${ }^{19}$ H. M. Mott-Smith and I. Langmuir, Phys. Rev. 28, 727 (1926).

${ }^{20}$ I. H. Hutchinson, Plasma Phys. Controlled Fusion 47, 71 (2005).

${ }^{21}$ M. S. Barnes, J. H. Keller, J. C. Forster, J. A. O'Neill, and D. K. Coultas, Phys. Rev. Lett. 68, 313 (1992).

${ }^{22}$ C. G. Granqvist and R. A. Buhrman, J. Appl. Phys. 47, 2200 (1976).

${ }^{23}$ T. Hihara and K. Sumiyama, J. Appl. Phys. 84, 5270 (1998).

${ }^{24}$ V. I. Kolobov and L. D. Tsendin, Plasma Sources Sci. Technol. 4, 551 (1995).

${ }^{25}$ V. Kouznetsov, K. Macák, J. M. Schneider, U. Helmersson, and I. Petrov, Surf. Coat. Technol. 122, 290 (1999).

${ }^{26} \mathrm{M}$. Hasan, "Modeling the extraction of sputtered species out of a pulsed hollow cathode," Master's thesis, XR-EE-SPP 2011:001 (Royal Institute of Technology, Sweden, 2011). 